\title{
Sexual Desire During the Menopausal Transition and Early Postmenopause: Observations from the Seattle Midlife Women's Health Study
}

\author{
Nancy Fugate Woods, R.N., Ph.D., Ellen Sullivan Mitchell, Ph.D., and Kathy Smith-Di Julio, Ph.D., R.N.
}

\begin{abstract}
Aims: To describe levels of sexual desire across the menopausal transition (MT) and early postmenopause (PM), including effects of age, MT-related factors, health, stress, symptoms (hot flash, sleep, mood), and social opportunity factors.

Methods: A subset of Seattle Midlife Women's Health Study (SMWHS) participants who provided data during the early reproductive, early and late menopausal transition stages, or postmenopause $(n=286)$, including menstrual calendars for staging the MT, annual health reports between 1990 and 2005, and morning urine samples assayed for estrone glucuronide $\left(\mathrm{E}_{1} \mathrm{G}\right)$, testosterone $(\mathrm{T})$, and follicle-stimulating hormone $(\mathrm{FSH})$ was included. Multilevel modeling using the $\mathrm{R}$ program was used to test factors related to sexual desire.

Results: Women experienced a significant decrease in sexual desire during the late MT stage $(p<0.01)$ and early PM $(p<0.0001)$. Those with higher urinary $\mathrm{E}_{1} \mathrm{G}$ and $\mathrm{T}$ reported significantly higher levels of sexual desire, whereas those with higher FSH levels reported significantly lower sexual desire $(p<0.0001,0.06$, and 0.0002 , respectively). Women using hormone therapy also reported higher sexual desire $(p=0.02)$. Those reporting higher perceived stress reported lower sexual desire $(p<0.0001)$, but history of sexual abuse did not have a significant effect. Those most troubled by symptoms of hot flashes, fatigue, depressed mood, anxiety, difficulty getting to sleep, early morning awakening, and awakening during the night also reported significantly lower sexual desire ( $p$ range from $<0.03$ to 0.0001 ), but there was no effect of vaginal dryness. Women with better perceived health reported higher sexual desire $(p<0.0001)$, and those reporting more exercise and more alcohol intake also reported greater sexual desire $(p<0.0001)$. Having a partner was associated with lower sexual desire. Conclusions: Clinicians working with women traversing the MT should be aware that promoting healthy sexual functioning among midlife women requires consideration of their changing biology as well as ongoing life challenges.
\end{abstract}

\section{Introduction}

$\mathbf{R}$ ECENT EFFORTS TO UNDERSTAND sexual response from women's perspectives have yielded new models of women's sexual desire, redefined as interest in sexual expression. ${ }^{1}$ This contrasts with earlier definitions of sexual desire focused on an urge for sexual activity produced by the activation of specific systems in the brain and experienced as a specific sensation that motivates an individual to seek or respond to sexual experience, now termed "libido." ${ }^{2}$ Basson et al. ${ }^{3,4}$ propose that women's sexual response occurs in a circular fashion, with overlapping phases instead of a linear progression through discrete phases, and that desire may occur spontaneously or may follow subjective arousal in re- sponse to sexual stimuli with an appropriate context instead of being present initially and triggering sexual expression. ${ }^{3}$ Women's awareness of sexual desire is not their most frequent reason for initiating or accepting sexual activity, ${ }^{4}$ and genital arousal does not correlate well with desire. ${ }^{3}$ Longitudinal data from the Study of Women's Health Across the Nation (SWAN) indicated that $24 \%$ of postmenopausal women reported they never felt and $41 \%$ infrequently felt sexual desire, but the majority reported experiencing arousal (92\%), and only $13 \%$ rated sex as not very important..$^{5}$ Thus, it is necessary to study sexual desire by asking women to rate their feelings of desire instead of using indirect measures.

To date, there is mixed evidence relating the menopausal transition (MT) to sexual desire. Participants in early studies

Department of Family and Child Nursing, University of Washington, Seattle, Washington. 
of sexuality and aging rarely attributed cessation of their sexual activity to a loss of desire or interest. Although postmenopausal women did note a lower level of interest in sex, multiple factors were involved, including death of a spouse, illness of a spouse, or their spouse's inability to perform sexual functions. ${ }^{6}$ In some studies, women who are in the MT or postmenopause (PM) report lower sexual interest or desire than do women who have not yet entered the MT, ${ }^{7-9}$ but in other study populations, there is no difference. ${ }^{10-13}$

Reports from longitudinal studies of women followed throughout the MP have contributed to a more thorough understanding of factors associated with sexual desire during the MT and early PM. Avis et al. ${ }^{14}$ found that postmenopausal women in the Massachusetts Women's Health Study reported a reduction in sexual desire more frequently than did women who had not yet begun the MT or were in the MT. Having had surgery (type not specified) during the past year was associated with an increase in sexual desire, but experiencing psychological symptoms, smoking, and having a partner were associated with lower sexual desire. ${ }^{14}$ Although Dennerstein et al. ${ }^{15}$ studied reports of libido measured as sexual thoughts or fantasies in the last month, in contrast to sexual desire, they found that women making a transition from the late reproductive stage or early MT to late MT did not experience a change in libido, but women changing from late MT to PM did report a decrease in libido. The findings of these studies suggest that factors related to the MT may influence sexual desire.

Recently published evidence suggests that endocrine markers of the MT, including estradiol $\left(E_{2}\right)$ and testosterone $(\mathrm{T})$, and use of exogenous hormone therapy may influence sexual desire. ${ }^{10,14,16,17}$ Avis et al. ${ }^{14}$ found that $E_{2}$ levels were not significantly related to sexual desire, but Dennerstein et al. ${ }^{15}$ reported marginal positive associations of $E_{2}$ and $T$ with sexual desire, and Cawood and Bancroft ${ }^{16}$ reported neither $\mathrm{E}_{2}$ nor $\mathrm{T}$ was associated. Santoro et al. ${ }^{17}$ found that $\mathrm{T}$ was associated minimally with increased sexual desire. Despite the inverse relationship between follicle-stimulating hormone (FSH) and $\mathrm{E}_{2}$ levels during the MT, to date there are no reports of relationships between FSH levels and sexual desire.

In addition to the physical changes associated with the MT, the context in which sexual desire occurs is influential. Perceived life stress has the potential to distract women from focusing on sexual desire, and a woman's social roles and responsibilities may limit her opportunity to engage with a partner in a situation conducive to sexual expression., $71,14,18-20$ Being married was associated with lower sexual desire compared with being single, divorced, or widowed. ${ }^{14,18}$ Role responsibilities that are linked to stress among midlife women include parenting as well as employment, and both may compete for women's time for sexual opportunity. Although history of sexual abuse has been linked to stress arousal and sexual dysfunction, it has not been studied in relation to sexual desire in midlife women.

Women experiencing such symptoms as hot flashes, mood, sleep disruption, fatigue, or vaginal dryness during the MT may find their symptoms are not conducive to sexual desire. ${ }^{15}$ Health-related behaviors, such as smoking, ingesting alcohol, and engaging in low levels of physical activity, have been associated with lower sexual desire in some studies. ${ }^{5,14,18}$ Experiencing poor health, including such chronic conditions that become more prevalent during the middle years as diabetes, depression, and cardiovascular disease (coronary artery disease), ${ }^{21-27}$ and medication use, including antihypertensives and antidepressants (SSRIs) may also be associated with reduced sexual desire during the MT and early $\mathrm{PM}^{28-32}$

Bancroft et al. ${ }^{33}$ found that although lower sexual desire increases with age, the proportion of women who are distressed about it decreases with age. These findings are consistent with those from the large cross-sectional study, Women's International Study of Health and Sexuality (WISHeS), in which women 50-70 years of age reported they were less distressed by their low sexual desire than were women $20-49$ years of age. ${ }^{34,35}$

Given the recent reconceptualization of women's sexual response, it is important to examine the consequences of the MT and aging for sexual desire, as well as the influence of health, stress, social opportunities, and symptoms on sexual desire. The purposes of this study were to:

1. Describe the level of sexual desire across the MT stages and early PM and with age.

2. Explore the relative associations between sexual desire and age, as they may be affected by MT factors (MT stage, urinary estrone glucuronide $\left[\mathrm{E}_{1} \mathrm{G}\right], \mathrm{FSH}, \mathrm{T}$, hormone therapy use), health-related factors (perceived health, smoking, alcohol intake, engaging in physical activity/exercising), social opportunity factors (being in a relationship with a partner, being a parent, being employed, years of education), stress-related factors (perceived stress, history of sexual abuse), and symptoms (hot flashes, depressed mood, anxiety, sleep disruption, fatigue, vaginal dryness).

\section{Materials and Methods}

\section{Design}

The data for these analyses are from a longitudinal study of the MT, the Seattle Midlife Women's Health Study (SMWHS). Women entered the cohort between 1990 and early 1992 when most were not yet in the MT or were in the early stages of the transition to menopause. After completing an initial in-person interview $(n=508)$ administered by a trained registered nurse interviewer, participants $(n=367)$ began providing data annually by questionnaire, menstrual calendar, and health diary. Also in 1990, women began contributing data in a health diary that included a symptom checklist as well as indicators of health behaviors and stress. Data were obtained from 3 days of diary ratings from those closest to days 5, 6, and 7 of the menstrual cycle and coinciding with first morning voided urine specimens women provided 8-12 times per year for endocrine assays (from late 1996 through 2000) and then quarterly for 2001-2005. These data were in addition to an annual health questionnaire and menstrual calendars.

\section{Sample}

Eligible participants $(n=286)$ were those who contributed ratings of sexual desire from the health diaries beginning in 1990 and were in the late reproductive stage, early or late MT stages, or early PM during the course of the study. The number of women providing data and the number of observations for each of the stages were: late reproductive $(n=195$, 2642 observations), early MT ( $n=169,1942$ observations), late 
MT ( $n=103,1059$ observations), early PM ( $n=65,974$ observations). Once they began the longitudinal component of the study, over $90 \%$ of eligible women participated each year. Women were eligible for participation if they had menstruated within the past 3 months before recruitment and had at least one ovary. Women using hormones were included, but their data were excluded from analyses reported here unless hormone therapy was included in the model being tested. They became ineligible once they attained 5 years PM or had a bilateral oophorectomy or induced menopause.

Women whose data were available for analysis and were eligible for inclusion were midlife women with a mean age of $41.4(\mathrm{SD}=4.3)$ years at the beginning of the study, 15.9 years of education $(\mathrm{SD}=2.8)$, and a median family income of $\$ 38,800$ $(\mathrm{SD}=\$ 15,000)$. Most $(87 \%)$ of the eligible participants were currently employed, $71 \%$ were married or partnered, $22 \%$ were divorced or separated or widowed, and $7 \%$ were never married or partnered. Eligible women described themselves as follows: 7\% African American, 9\% Asian American, 82\% Caucasian. As seen in Table 1, women who were included in these analyses compared with those who were ineligible were similar with respect to employment status, marital status, age, and education. They differed significantly by income and ethnicity: those who were included in the analyses had higher incomes and were more likely to be Caucasian.

\section{Measures}

The measures used for this study included sexual desire, age, MT-related factors (MT stage, urinary $E_{1} G, F S H$, and T; hormone therapy use), stress-related factors (perceived stress, history of sexual abuse), symptoms (hot flashes, depressed mood, anxiety, sleep disruption, fatigue, vaginal dryness), social opportunity factors (having a partner, being a parent or employed, years of education), and health-related factors (perceived health, using alcohol, smoking, exercising).

Sexual desire. Sexual desire was assessed in the diary by asking women to respond to the item: Rate how much you felt sexual desire: $0=$ not at all, $1=$ minimally, $2=$ mildly, $3=$ moderately, and $4=$ very much. The reference period for sexual desire ratings was the past 24 hours. Because sexual desire is a state, an estimate for a 24 -hour period was optimal.

Menopausal transition-related factors. MT-related factors included MT stage, urinary $E_{1} G, T, F S H$, and hormone therapy use. Hormone therapy use did not include oral contraceptives or progestin alone. Using menstrual calendar data, women not taking any hormones were classified according to stages of reproductive aging: late reproductive, early MT, late MT, or early PM, based on staging criteria developed by Mitchell et al. $^{36}$ The names of stages match those recommended at the Stages of Reproductive Aging Workshop (STRAW). ${ }^{37}$ The time before the onset of persistent menstrual irregularity during midlife was labeled the late reproductive stage when cycles were regular. Early stage was defined as persistent irregularity of $>6$ days absolute difference between any two consecutive menstrual cycles during the calendar year, with no skipped periods. Late transition stage was defined as persistent skipping of one or more menstrual periods. A skipped period was defined as double the modal cycle length or more for the calendar year. In the absence of a modal cycle length, a population-based cycle length of 29 days was used. Persistence meant the event, irregular cycle or skipped period, occurred one or more times in the subsequent 12 months. Final menstrual period (FMP) was identified retrospectively after 1 year of amenorrhea without any known explanation. The date of the FMP is synonymous with the term menopause. Early PM was within 5 years of the FMP. The criteria for staging the MT using these definitions have been described in detail elsewhere and have been validated in the ReSTAGE Study. ${ }^{38}$ Women who were using hormone therapy at recruitment were excluded from the study. Those using hormone therapy in any period could not be assigned an MT stage because their bleeding or lack of bleeding prevented assigning an MT stage, applying the staging rules that were developed for women experiencing a natural menopause.

Table 1. Sample Characteristics of Eligible and Ineligible Women in the Mixed Effects Modeling Analyses of Sexual Desire at Start of Study, 1990-1991

\begin{tabular}{lccc}
\hline Characteristic & Eligible women $(\mathrm{n}=286)$ Mean $(S D)$ & Ineligible women $(\mathrm{n}=222)$ Mean $(S D)$ & $\mathrm{p}$ value \\
\hline Age, years & $41.4(4.3)$ & $42.1(5.0)$ & $15.4(3.1)$ \\
Years of education & $15.9(2.8)$ & $\$ 35,600(\$ 17,400)$ & 0.09 \\
Family income & $\$ 38,800(\$ 15,000)$ & $n(\%)$ & 0.07 \\
& $n(\%)$ & $189(85.1)$ \\
Currently employed & & $33(14.9$ & 0.53 \\
Yes & $249(87.1)$ & $38(17.1)$ \\
No & $37(12.9)$ & $17(7.7)$ \\
Race/ethnicity & $20(7.0)$ & $156(70.3)$ \\
African American & $26(9.1)$ & $11(4.9)$ \\
Asian/Pacific Islander & $235(82.2)$ & $145(65.3)$ \\
Caucasian & $5(1.7)$ & $62(27.9)$ \\
Other (Hispanic, mixed) & & $15(6.8)$ \\
Marital status & $203(71.0)$ & $<.0001$ \\
Married/partnered & $63(22.0)$ & 0.31 \\
Divorced/widowed/not partnered & $20(7.0)$ & \\
Never married/partnered & & \\
\hline
\end{tabular}

\footnotetext{
${ }^{\mathrm{a}}$ Independent $t$ test.
}

${ }^{\mathrm{b}}$ Chi-square test. 
Urinary assays. Urinary assays were performed in our laboratories using a first-voided morning urine specimen provided on day 6 of the menstrual cycle, if menstrual periods were identifiable. For women with no bleeding or spotting or extremely erratic flow, a consistent date each month was used. Women abstained from smoking, caffeine use, and exercise before the urine collection. Urine samples were preserved with sodium ethylenediamine tetraacectic acid and sodium metabisulfite and frozen at $-70^{\circ} \mathrm{C}$. All specimens, standards, and controls were tested in duplicate, and those with a coefficient of variance $>15 \%$ were repeated. A Bio-Rad Quantitative Urine control (Bio-Rad, Hercules, CA) and a pooled in-house urine control were included in all assays, and a member of the standard curve was repeated after every ten unknowns to monitor assay performance. In general, all samples from a calendar year were assayed during the next calendar year, and multiple samples from each participant were assayed in the same batch during each year. All endocrine concentrations were corrected for variations in urine concentration by expressing the hormone level concentration ratio in the same urine specimen.

Urinary $E_{1} G$ was selected to assess estrogens because it is stable, can be reliably measured without special preparation, and is highly correlated with serum $E_{2}$ levels. ${ }^{39-44}$ Urinary $\mathrm{E}_{1} \mathrm{G}$ was measured by a competitive enzyme immunoassay (EIA) that cross-reacts $83 \%$ with $E_{1} G$ and $<10 \%$ with free $E_{1}$, estrone sulfate, estriol glucuronide, $\mathrm{E}_{2}$, and estriol. ${ }^{41}$ The assay is described in detail elsewhere. ${ }^{41}$ The lower limit of detection for the assay was $3.1 \mathrm{nmol} / \mathrm{L}$. Average recovery from a urine matrix of low, medium, and high $\mathrm{E}_{1} \mathrm{G}$ standard doses was $101 \%{ }^{41}$ Intra-assay and interassay coefficients of variation $(\mathrm{CV})$ were $2.1 \%$ and $9.6 \%$, respectively, for an external (BioRad) urine control (mean concentration $2.1 \mathrm{ng} / \mathrm{mL}$ ) and $2.8 \%$ and $14.5 \%$, respectively, for an internal urine control (mean concentration $1.59 \mathrm{ng} / \mathrm{mL}$ ) (determined using the method of Robard $^{45}$ from 20 randomly selected plates).

Urinary FSH was assayed using Diagnostic Products Corporation (DPC, Los Angeles, CA) Double Antibody FSH Kit, using a radioimmunoassay (RIA) designed for the quantitative measurement of FSH in serum and urine. The procedure is described in detail elsewhere. ${ }^{46}$ The reporting range for urine FSH was $2.0-100 \mathrm{mIU} / \mathrm{mL}$; the minimum detectable concentration was $1.6 \mathrm{mIU}$. The interassay variation was $7.1 \%$, and the intra-assay variation was $3.7 \%(n=205)$.

Urinary creatinine was assayed in urine specimens using the method of Jaffe. ${ }^{47}$ The interassay variation (run to run) was $6.7 \%$, and the intra-assay variation was $3.1 \%(n=405)$. Urinary $\mathrm{T}$ levels were assayed using the Siemens Total Testosterone Kit (Siemens, Deerfield, IL), a solid-phase RIA using a testosterone-specific antibody immobilized to the wall of a polypropylene tube. The assay is described in detail elsewhere. ${ }^{46}$ The interassay variation was $12.38 \%(n=791)$, and the intra-assay variation was $8.75 \%$.

Health-related factors. Health-related factors included perceived health, smoking, using alcohol, and exercising. Perceived health was measured in the diary from the beginning of the study using the question: How healthy did you feel today? Women rated their perceived health from 1 (not at all) to 6 (extremely, a lot).

Women were asked to indicate in the daily health diary whether or not they smoked (coded as 0 for no and 1 for yes), the amount of alcohol drunk, and the amount they exercised. Exercise was determined using the question: How many total minutes of nonwork-related exercise did you do today? This includes walking, running, biking, swimming, aerobics, sports, work out, gardening, and yard work.

Medication use was coded from the health diaries and included medications that fell into categories, such as androgens, antidepressants, hormonal preparations, SERMS, sedatives, and antipsychotics. These variables were used to exclude data points from the analyses where medication use would be likely to confound the relationship being tested. Use was coded 1 for yes or 0 for no for each time point.

Stress-related factors. Stress-related factors included perceived stress and history of sexual abuse. Perceived stress was assessed in the diary with a question: How stressful was your day? Women rated their responses from 0 (not at all) to 6 (extremely, a lot). Brantley et al. ${ }^{48}$ found that a global stress rating and the sum of stress ratings across multiple dimensions correlated significantly $(r=0.35, p<0.01)$. Sexual abuse history was assessed by asking: Have you ever been sexually assaulted, abused, or molested? These data were obtained in 1999-2002 in the annual health update questionnaire. Also, beginning in 1996 and through the end of the study, we asked: During the past year, did you experience any sexual abuse or sexual assault? A cumulative variable was created to represent any history of sexual abuse or assault and coded as 1 for yes and 0 for no.

Social opportunity factors. Social opportunity factors were assessed annually in the health report and included whether the woman had a partner, was employed, and was a parent, with each variable coded as 1 for yes and 0 for no. In addition, education was assessed annually as years of education completed.

Symptoms. Hot flash severity was assessed several times each year in the symptom diary, in which women rated their symptoms from 0 (not present) to 4 (extreme). Depressed mood (feeling sad or blue), anxiety, difficulty getting to sleep, awakening during the night, early morning awakening, fatigue, and vaginal dryness were assessed in a similar fashion.

\section{Analyses}

Mixed effects modeling using the R library ${ }^{49-53}$ was used to investigate if age, MT stage, factors related to the MT, stressrelated factors, symptoms, social factors, and health-related factors were significant predictors of sexual desire over time. Age was centered at the group mean to enable interpretation of the effect of age on sexual desire. Details of the analytic models are provided in detail elsewhere. ${ }^{46}$

The initial series of models tested age alone as a predictor of sexual desire. The first model postulated that overall levels of sexual desire could differ from woman to woman (random intercept), but the scores would change with age in a common manner (fixed slope). The second model extended the first to postulate that each woman has a different mean level of sexual desire and rate of change (random intercept, random slope). The best fitting model was assessed by using maximum likelihood estimation with Akaike Information Criterion (AIC). ${ }^{53}$ When the best fitting model was found, we 
Table 2. Random Effects Models for Sexual Desire with Age as Predictor $\left(\beta_{2}\right)$ AND WITH INDIVIDUAL COVARIATES $\left(\beta_{3}\right)^{\mathrm{a}}$

\begin{tabular}{|c|c|c|c|c|c|c|c|c|}
\hline \multirow[b]{2}{*}{ Predictor } & \multicolumn{3}{|c|}{ Mean values ${ }^{\mathrm{b}}$ ( $\mathrm{p}$ values) } & \multicolumn{3}{|c|}{ Standard deviations ${ }^{c}$} & \multicolumn{2}{|c|}{ Number } \\
\hline & $\beta_{1}{ }^{\mathrm{b}}$ & $\beta_{2}{ }^{b}$ & $\beta_{3}^{* *}$ & $\sigma_{1}^{* * *}$ & $\sigma_{2}^{* * *}$ & $\sigma_{\varepsilon}^{* * *}$ & Women & Observations \\
\hline Age (47.3) & 1.26 & $0.02(0.04)$ & - & 0.87 & 0.09 & 0.69 & 286 & 6617 \\
\hline \multicolumn{9}{|l|}{ Health-related factors } \\
\hline Perceived health & $0.42(<0.0001)$ & $0.02(0.04)$ & $0.21(<0.0001)$ & 0.860 & 0.093 & 0.674 & 286 & 6617 \\
\hline If smokes & $1.28(<0.0001)$ & $0.01(0.05)$ & $-0.16(0.03)$ & 0.87 & 0.09 & 0.69 & 286 & 6617 \\
\hline Amount of exercise & $1.20(<0.0001)$ & $0.01(0.05)$ & $0.002(<0.0001)$ & 0.87 & 0.09 & 0.69 & 286 & 6617 \\
\hline Amount of alcohol & $1.20(<0.0001)$ & $0.01(0.05)$ & $0.10(<0.0001)$ & 0.86 & 0.09 & 0.69 & 286 & 6617 \\
\hline \multicolumn{9}{|c|}{ Menopausal transition factors } \\
\hline MT stage & $1.31(<0.0001)$ & $0.03(0.0001)$ & & 0.87 & 0.08 & 0.69 & 286 & 6617 \\
\hline Early & & & $0.02(0.54)$ & & & & & \\
\hline Late & & & $-0.13(0.01)$ & & & & & \\
\hline Early PM & & & $-0.42(<0.0001)$ & & & & & \\
\hline $\begin{array}{l}\text { Estrone glucuronide } \\
\quad\left(\log _{10}\right)(1.3)\end{array}$ & $1.55(<0.0001)$ & $-0.03(0.003)$ & $0.21(<0.0001)$ & 0.92 & 0.09 & 0.62 & 130 & 4718 \\
\hline $\mathrm{FSH}\left(\log _{10}\right)(1.1)$ & $1.54(<0.0001)$ & $-0.03(0.002)$ & $-0.06(0.006)$ & 0.93 & 0.09 & 0.62 & 130 & 4751 \\
\hline Testosterone $\left(\log _{10}\right)(1.2)$ & $1.52(<0.0001)$ & $-0.04(0.001)$ & $0.15(0.0002)$ & 0.92 & 0.09 & 0.63 & 130 & 4754 \\
\hline HRT use & $1.23(<0.0001)$ & $0.01(0.10)$ & $0.10(0.02)$ & 0.85 & 0.09 & 0.69 & 312 & 7566 \\
\hline \multicolumn{9}{|l|}{ Social opportunity factors } \\
\hline If partnered & $1.34(<0.0001)$ & $0.02(0.04)$ & $-0.12(0.008)$ & 0.87 & 0.09 & 0.69 & 286 & 6617 \\
\hline Years of education & $0.77(0.01)$ & $0.02(0.04)$ & $0.03(0.12)$ & 0.87 & 0.09 & 0.69 & 286 & 6617 \\
\hline If parent & $1.23(<0.0001)$ & $0.01(0.04)$ & $0.03(0.72)$ & 0.87 & 0.09 & 0.69 & 286 & 6617 \\
\hline Employment & $1.28(<0.0001)$ & $0.02(0.04)$ & $-0.02(0.75)$ & 0.87 & 0.09 & 0.69 & 286 & 6617 \\
\hline \multicolumn{9}{|l|}{ Stress-related factors } \\
\hline Perceived stress & $1.50(<0.0001)$ & $0.01(0.12)$ & $-0.10(<0.0001)$ & 0.88 & 0.09 & 0.69 & 286 & 6617 \\
\hline History of sexual abuse & $1.23(<0.0001)$ & $0.02(0.02)$ & $0.21(0.09)$ & 0.89 & 0.09 & 0.69 & 231 & 6415 \\
\hline \multicolumn{9}{|l|}{ Symptoms } \\
\hline Hot flashes & $1.27(<0.0001)$ & $0.02(0.01)$ & $-0.05(0.0002)$ & 0.87 & 0.09 & 0.69 & 286 & 6612 \\
\hline Fatigue & $1.46(<0.0001)$ & $0.02(0.03)$ & $-0.17(<0.0001)$ & 0.88 & 0.09 & 0.68 & 286 & 6617 \\
\hline Depressed mood & $1.34(<0.0001)$ & $0.02(0.03)$ & $-0.20(<0.0001)$ & 0.90 & 0.10 & 0.66 & 286 & 5992 \\
\hline Anxiety & $1.34(<0.0001)$ & $0.02(0.02)$ & $-0.10(<0.0001)$ & 0.89 & 0.09 & 0.69 & 286 & 6458 \\
\hline Problem getting to sleep & $1.31(<0.0001)$ & $0.02(0.02)$ & $-0.13(<0.0001)$ & 0.89 & 0.09 & 0.69 & 286 & 6458 \\
\hline $\begin{array}{l}\text { Awakening during } \\
\text { the night }\end{array}$ & $1.30(<0.0001)$ & $0.02(0.01)$ & $-0.06(<0.0001)$ & 0.88 & 0.09 & 0.69 & 286 & 6458 \\
\hline Early morning awakening & $1.28(<0.0001)$ & $0.02(0.02)$ & $-0.03(0.03)$ & 0.88 & 0.09 & 0.69 & 286 & 6458 \\
\hline Vaginal dryness & $1.26(<0.0001)$ & $0.02(0.03)$ & $-0.03(0.20)$ & 0.87 & 0.09 & 0.69 & 286 & 6617 \\
\hline
\end{tabular}

${ }^{a}$ Occasions on which selected medications were used were omitted from the analyses. Androgen use occasions were excluded from all analyses. Occasions when medications could affect symptoms, such as sleep, hot flashes, and mood, were excluded from these analyses.

${ }^{\mathrm{b}} \beta_{1}, \beta_{2}, \beta_{3}$ are the fixed effects (group averages) for the intercept, slope, and covariate.

${ }^{c} \sigma_{1}, \sigma_{2}, \sigma_{\varepsilon}$ are the random effects (variability) for the intercept, slope and residual error.

extended that model by adding covariates iteratively to test the effect on sexual desire scores over time. The next step was to determine the best model for each concept by adding all significant covariates within each concept. Next, all covariates that significantly improved the model fit to the data when entered by concept were added simultaneously into a final model. Finally, a reduced final model was considered using only the significant variables from the final model. A $p$ value of 0.05 was used as the criterion for significance. Different numbers of women and observations occurred with each variable tested because the analysis required pairing of observations of the outcome and predictor variables at each time point. For example, in cases of medication use that could confound the results, data were omitted from analyses for that occasion.

\section{Results}

Results in Table 2 describe all six model parameters. The intercept $\left(\beta_{1}\right)$ is the initial or baseline mean value for sexual desire at age 47.3 for this population and has significant variability ( $p=<0.0001)$. Age was centered at 47.3 years, the mean for the women whose data were used in the following analyses, to enhance interpretation of the effect of age on sexual desire. When age effects on sexual desire were analyzed using a random intercept, fixed slope model vs. a random intercept, random slope model, the latter provided a significantly better fit to the data (AIC 15393.33 vs. 15030.45, $p=<0.0001$ ). When age was considered alone using a random effects model, at age 47.3 , the mean sexual desire level was 1.26 , with a small but significant increase of 0.02 per year $\left(\beta_{2}\right)$ (Table 2$)$.

The slope $\left(\beta_{2}\right)$ is the mean increase or decrease in sexual desire per year, and the covariate value $\left(\beta_{3}\right)$ is the mean increase or decrease from the intercept in sexual desire with each unit of change in the covariate. For example, the perceived health response range is from 1 to 10 . With perceived health as a covariate with age in the equation, the mean sexual desire level at age 47.3 for this population at the start of the study $\left(\beta_{1}\right)$ was 0.42 . Sexual desire increased by 0.02 units $\left(\beta_{2}\right)$ 
Level of Sexual Desire by MT Stage

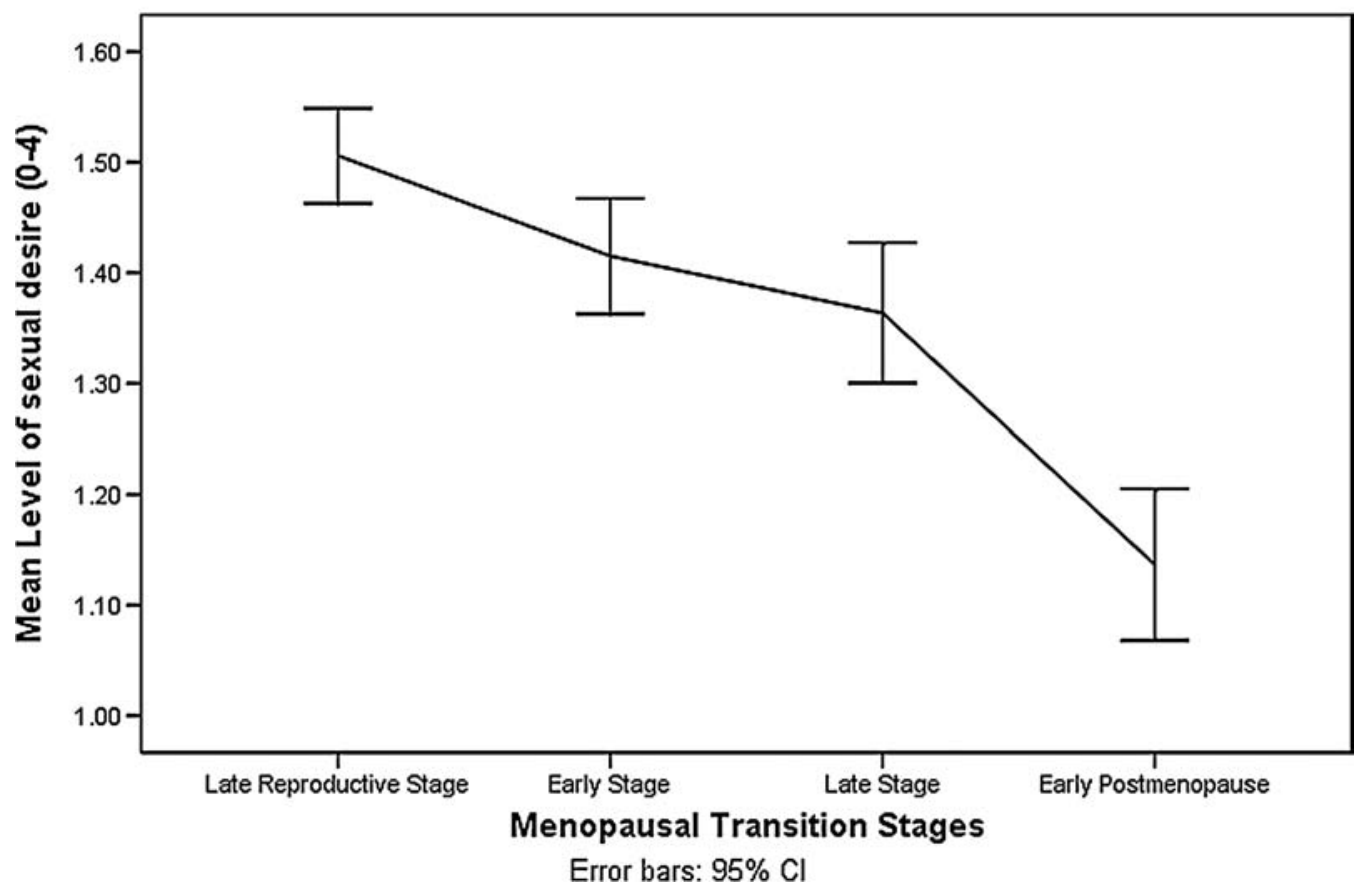

FIG. 1. Sexual desire by menopausal transition stage.

per year during the study, and for every 1 unit increase in perceived health, sexual desire significantly increased by 0.21 units $\left(\beta_{3}\right)$.

As seen in Table 2, the health-related covariates all had a significant effect on sexual desire when added individually. Perceived postiive health predicted a significant increase in sexual desire $(\beta=0.21, p=<0.0001)$. Amount of exercise and alcohol intake also predicted a significant increase in sexual desire ( $\beta=0.002$ and 0.10 , respectively, $p=<0.0001$ for both), and smoking status predicted a significant decrease in sexual desire $(\beta=-0.16, p=0.03)$.

MT-related covariates (MT stage, $\mathrm{E}_{2}, \mathrm{FSH}, \mathrm{T}$, and use of hormone therapy) were analyzed with age as a covariate in the model using a random effects model. For this population of women, those in the late MT and early PM experienced significantly lower sexual desire than those in the late reproductive stage $(\beta=-0.13, p=0.01$ for late MT; $\beta=-0.42$, $p=<0.0001$ for early PM) (Table 2 ). As seen in Figure 1, being in the early MT stage had little effect on sexual desire compared with being in the late reproductive stage. The steepest drop in sexual desire occurred between late MT stage and early PM. The level of sexual desire decreased gradually with years prior to the final menstrual period, with the steepest drop from 3 years before FMP to 2 years after FMP (Fig. 2).

Of the endocrine factors studied with age as a covariate, $\mathrm{E}_{2}$ was positively related to sexual desire $(\beta=0.21, p=<0.0001)$, as was $\mathrm{T}(\beta=0.15, p=<0.0002)$. FSH was negatively related to sexual desire $(\beta=-0.06, p=0.006)$, and hormone therapy use was positively related to sexual desire $(\beta=0.10, p=0.02)$.

When social opportunity covariates were added to a model with age as a covariate, having a partner was associated with a decrease in sexual desire $(\beta=-0.12, p=0.008)$. Other social opportunity factors, including parenting, employment, and years of education had no significant effect on sexual desire.
For the stress-related covariates when analyzed with age as a covariate, women who reported greater perceived stress experienced significantly lower levels of sexual desire $(\beta=-0.10, p=<0.0001)$. History of sexual abuse had no significant effect on sexual desire.

Severity of symptoms women reported had a significant negative effect on sexual desire. Among these, hot flashes $(\beta=-0.05, p=0.0002)$, anxiety $(\beta=-0.10, p=<0.0001)$, difficulty getting to sleep $(\beta=-0.13, p=<0.0001)$, awakening during the night $(\beta=-0.06, p=<0.0001)$, awakening early in the morning $(\beta=-0.03, p=0.03)$, fatigue $(\beta=-0.17$, $p=<0.0001)$, and depressed $\operatorname{mood}(\beta=-0.20, p=<0.0001)$ were associated with lower sexual desire. Vaginal dryness was not related to sexual desire.

When all significant covariates for all concepts were analyzed together in a final model, covariates with a significant effect on sexual desire included age, $E_{1}, T$, perceived health, amount of alcohol intake, perceived stress, $E_{1}$, depressed mood, fatigue, and problem getting to sleep (Table 3). Of these, age, perceived health, stress, depressed mood, and fatigue had significant effects, with $p<0.0001$. The effect of $\mathrm{T}$ $(p=0.01)$ was considered marginal in this model.

\section{Discussion}

As women in the SMWHS cohort aged, their reported levels of sexual desire decreased only slightly. MT factors, health-related factors, stress, and symptoms had important effects on desire, but social opportunity factors had limited effects.

Sexual desire began to drop during the late MT stage and continued to drop more markedly in the early PM. As estrogen and T levels dropped and FSH levels rose, sexual desire decreased. Hormone therapy use was associated with slightly 
Level of Sexual Desire

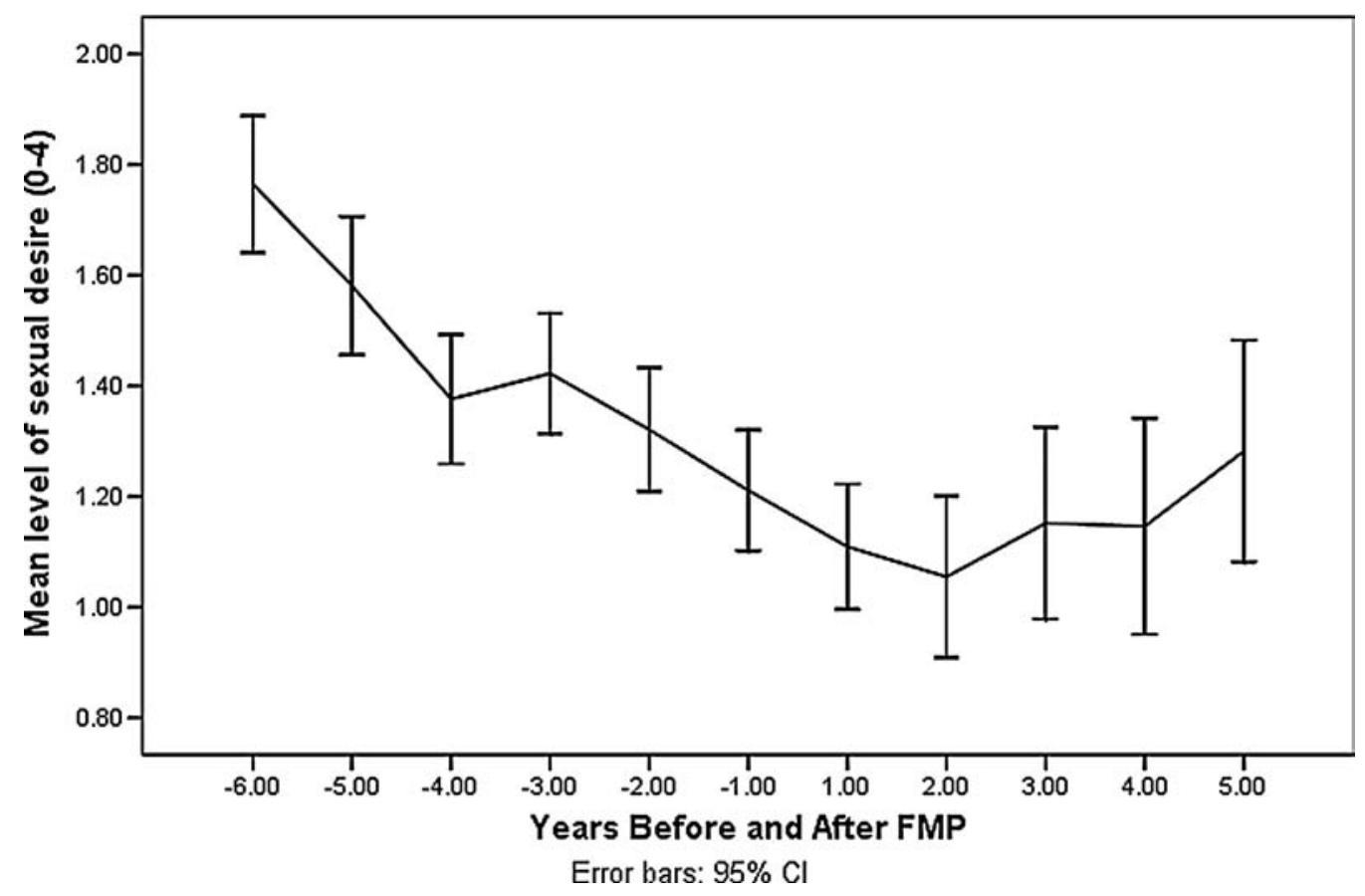

FIG. 2. Sexual desire by years before and after final menstrual period.

higher levels of sexual desire. Women who reported better perceived health and those who reported lower perceived stress levels, higher levels of exercise, and more use of alcohol reported higher levels of sexual desire. Women who experienced more severe symptoms, including hot flashes, fatigue, depressed mood, anxiety, problems getting to sleep, early morning awakening, and awakening during the night, reported lower sexual desire, but there was not a significant

Table 3. Final Random Effects Model for Sexual Desire, with Age as Predictor and Other Significant Covariates Entered Simultaneously ${ }^{\mathrm{a}}$

\begin{tabular}{lccc}
\hline & \multicolumn{3}{c}{$\begin{array}{c}\text { Standard } \\
\text { Error }\end{array}$} \\
& $\begin{array}{c}\text { Coefficient } \\
(\beta)\end{array}$ & $\begin{array}{c}\text { Standard } \\
\text { Deviation }\end{array}$ & p value \\
\hline Fixed effects & & & \\
$\beta_{1}$ intercept & 1.18 & 0.11 & $<0.0001$ \\
$\beta_{2}$ Age $(-47.3)$ years & -0.05 & 0.01 & $<0.0001$ \\
$\beta_{3}$ E $\mathrm{G}$ & 0.14 & 0.04 & $<0.0001$ \\
$\beta_{4}$ Testosterone & 0.11 & 0.04 & 0.01 \\
$\beta_{5}$ Perceived health & 0.16 & 0.01 & $<0.0001$ \\
$\beta_{6}$ Alcohol drinks & 0.06 & 0.02 & 0.0007 \\
$\beta_{7}$ Stress & -0.05 & 0.01 & $<0.0001$ \\
$\beta_{8}$ Depressed mood & -0.13 & 0.02 & $<0.0001$ \\
$\beta_{9}$ Fatigue & -0.08 & 0.01 & $<0.0001$ \\
$\beta_{10}$ Problems & -0.06 & 0.02 & 0.001 \\
going to sleep & & & \\
Random effects & & 0.10 & \\
$\beta_{1}$ Intercept $\sigma_{1}$ & & 0.09 & \\
$\beta_{2}$ Age $(-47.3)$ years $\sigma_{2}$ & & 0.56 & \\
$\beta_{\varepsilon}$ residual $\sigma_{\varepsilon}$ & & & \\
\hline
\end{tabular}

${ }^{\mathrm{a}} n=126 ;$ observations $=4086$. effect of vaginal dryness. None of the factors indexing social opportunity, except having a partner and education, were significantly related to sexual desire.

The most parsimonious model obtained by multivariate analyses included age, which was negatively associated with sexual desire, and both $E_{1}$ and $T$, which were positively related to sexual desire. In addition, perceived health and alcohol intake were both positively associated with sexual desire, and perceived stress, depressed mood, fatigue, and problems getting to sleep were associated negatively with sexual desire.

Our findings of a positive relationship between urinary $E_{1} G$ and sexual desire are consistent with reports by others who have assayed $E_{2}$ levels in serum and identified a positive association with sexual desire. ${ }^{10}$ In addition, the use of hormone therapy was positively associated with sexual desire, suggesting that both endogenous and exogenous estrogen sources may be important in promoting/supporting sexual desire. Our finding of a positive relationship between urinary $\mathrm{T}$ levels and sexual desire is consistent with the SWAN study results, ${ }^{17}$ as well as the findings of Gracia et al. ${ }^{54}$ of an association between fluctuating levels of $\mathrm{T}$ (vs. stable) and decreased libiodo. The relationship of FSH to sexual desire we found in the SMWHS cohort was not reported in prior studies. Although FSH may not have a direct central effect on perception of sexual desire, it may serve as a marker for decreasing $E_{2}$ levels during progression through the MT. Of note is that only $E_{1} G$ and $\mathrm{T}$ were significant in the multivariate model and the $p$ value for $\mathrm{T}$ was much larger than that for $\mathrm{E}_{1} \mathrm{G}$ and considered marginally significant given the number of tests.

The relationship of sexual desire to the progression through MT stages, particularly becoming postmenopausal, is consistent with findings of other studies in which a decrease in sexual desire was seen as women made the transition from the 
late reproductive stage to the MT stages or early PM. ${ }^{14,15,55}$ Although there was no association between MT stages and sexual desire in the SWAN baseline measures, few women were in the late MT stage and none were in early PM at the time of the measures. ${ }^{56}$ In our multivariate model, MT stages did not have significant effects on sexual desire.

Experiencing symptoms of hot flashes, fatigue, depressed mood, anxiety, and sleep problems were all associated with reduced levels of sexual desire. Dennerstein et al. ${ }^{15}$ also found that women most troubled by symptoms during the MT or PM experienced lower sexual desire, as did Reed et al. ${ }^{57}$ In particular, hot flashes, night sweats, disrupted or poor sleep, and depressed mood were frequently reported correlates for decreased sexual desire. ${ }^{54,57}$ Unlike studies of other populations, we found no relationship with vaginal dryness and sexual desire. ${ }^{54,56,57}$ These findings may reflect our focus on assessing sexual desire vs. libido. Other studies assessing sexual desire include items referencing libido and, thus, may be detecting perceptions of vaginal sensations of lubrication and swelling, which may be influenced by symptoms such as vaginal dryness. The important effects of symptoms were underscored in the multivariate model, in which depressed mood, fatigue, and problems getting to sleep were all significant.

Stress-related factors, including higher perceived stress levels, were associated with lower sexual desire, as seen in other studies. ${ }^{18-20,56}$ This relationship is consistent with inability to focus on oneself when the environmental demands challenge safety and intimacy. Of interest is that history of sexual abuse was not associated with sexual desire during midlife, similar to that found in the SWAN cohort baseline measures. ${ }^{56}$ Perceived stress was the only stress-related factor that was significant in the multivariate model.

Having a partner was negatively associated with sexual desire, consistent with results from the baseline assessment of sexual desire in the SWAN cohort as negatively related to being married, suggesting that relationship novelty may be a factor in sustaining sexual desire. ${ }^{56,58}$ This explanation would be consistent with results of a postal survey of Australian women indicating that length of relationship was associated with lower sexual desire. ${ }^{58}$

Aside from having a partner, social opportunity factors, including role obligations (parent, employment), that may be burdensome and education were unrelated to sexual desire, in contrast to their effects on sexuality in other studies. ${ }^{15,18-20}$ Education may not have an apparent effect in the SMWHS cohort, who were well-educated. Likewise, parenting and employment effects may not have been significant given the ages of adult children and the relatively high proportion of employment in the cohort. None of the social factors was significant in the multivariate model.

Health-related factors were significantly associated with sexual desire. Women reporting more positive perceptions of their health reported greater sexual desire, consistent with findings in other studies. ${ }^{14}$ Women who engaged in physical exercise and used alcohol also reported greater sexual desire, consistent with results of other studies. ${ }^{12,14,19}$ Our finding of a negative relationship between smoking and sexual desire has not been reported previously and may be related to effects of smoking on estrogen levels during the menopausal transition. Only perceived health and alcohol use were significant in the multivariate model.
Taken together, these findings show a profile of vulnerability to reduced sexual desire associated with aging, lower estrogen and $\mathrm{T}$ levels, poorer perceived health, lower alcohol use, exposure to stress, and experience of depressed mood, fatigue, and problems getting to sleep. Future attention to both biological and psychosocial correlates of sexual desire, including everyday stress and symptoms women may experience in conjunction with the MT, is warranted.

The limitations of this study include restriction of the sexual desire ratings to days 5-7 of the menstrual cycle for cycling women, during the early follicular phase. In addition, women who were using hormone therapy at the beginning of the study were excluded. Those who used hormone therapy later during some observations were included in the analyses reported there, but observations made during hormone therapy use were omitted from the analyses except for the analysis of their effects (Table 2). Finally, women who withdrew from the study before completing the first 5 years of the PM reduced the numbers of women providing data for these observations. Future studies of sexual desire during the MT and PM will be necessary in order to reveal a longer course of sexual desire as women experience the late PM and age.

\section{Acknowledgments}

This research was supported in part by grants from the National Institute of Nursing Research, P50-NU02323, P30NR04001, and R01-NR0414. We acknowledge the contributions of Don Percival, who designed the analytic strategies for this article.

\section{Disclosure Statement}

The authors have no conflicts of interest to report.

\section{References}

1. Basson R. The female sexual response: A different model. J Sex Marital Ther 2000;26:51-65.

2. Kaplan H. Disorders of sexual desire. New York: BrunnerMazel, 1979.

3. Basson R. Hormones and sexuality: Current complexities and future directions. Maturitas 2007;57:66-70.

4. Basson R, Leiblum S, Brotto L, et al. Revised definitions of women's sexual dysfunction. J Sex Med 2004;1:40-48.

5. Avis NE, Brockwell S, Randolph J, et al. Longitudinal change in sexual functioning as women transition through menpause: Results from the Study of Women's Health Across the Nation. Menopause 2009;16:442-452.

6. Pfeiffer E, Verwoerdt A, Davis G. Sexual behavior in middle life. Am J Psychiatry 1972;128:1262-1267.

7. Hallstrom T. Sexuality in the climacteric. Clin Obstet Gynecol 1977;4:227-239.

8. Hunter M, Battersby, R, Whitehead M. Relationships between psychological symptoms, somatic complaints, and menopausal status. Maturitas 1986;8:217-228.

9. Dennerstein L, Smith A, Morse C, Burger J. Sexuality and the menopause. J Psychosom Obstet Gynecol 1994;15:59-66.

10. Dennerstein L, Dudley E, Hopper J, Burger H. Sexuality, hormones, and the menopausal transition. Maturitas 1997;26: 83-93.

11. Hawton K, Gath D, and Day A. Sexual function in a community sample of middle-aged women with partners: Effects of age, marital, socioeconomic, psychiatric, gynecological, and menopausal factors. Arch Sex Behav 1994;23:375-395. 
12. Koster A, Garde K. Sexual desire and menopausal development. A prospective study of Danish women born in 1936. Maturitas 1993;16:49-60.

13. Osborn M, Hawton K, Gath D. Sexual dysfunction among middle aged women in the community. BMJ 1988;296:959962.

14. Avis N, Stellato M, Crawford S, Johannes C, Longcope C. Is there an association between menopause status and sexual functioning? Menopause 2000;7:297-309.

15. Dennerstein L, Dudley E, Burger H. Are changes in sexual functioning during midlife due to aging or menopause? Fertil Steril 2001;76:456-460.

16. Cawood E, Bancroft J. Steroid hormones, the menopause, sexuality and well-being of women. Psychol Med 1996;26: 925-936.

17. Santoro N, Torrens J, Crawoford S, et al. Correlates of circulating androgens in midlife women: The study of women's Health Across the Nation. J Clin Endocrinol Metab 2005; 90:4836-4845.

18. Greendale G, Hogan P, Shumaker S. Sexual functioning in postmenopausal women: The Postmenopausal Estrogen/ progestin Interventions (PEPI) Trial. J Womens Health 1996; 5:445-458.

19. Mansfield P, Koch P, Voda A. Midlife women's attributions for their sexual response changes. Health Care Women Int 2000;21:543-559.

20. Dennerstein L, Smith A, Morse C. Psychological well-being, mid-life, and the menopause. Maturitas 1994;20:1-11.

21. Enzlin P, Mathieu C, Ban den Vruel A, Bosteels J, Vanderschueren D, Demyttenaere K. Sexual dysfunction in women with type 1 diabetes: A controlled study. Diabetes Care 2002;25:672-677.

22. Erol B, Tefekli A, Ozbey I, et al. Sexual dysfunction in type II diabetic females: A comparative study. J Sex Marital Ther 2002;28(Suppl 1):55-62.

23. Coyle C, Santiago M, Shank J, Ma G, Boyd R. Secondary conditions and women with physical disabilities: A descriptive study. Arch Phys Med Rehabil 2000;81:1380-1387.

24. Dunn K, Croft P, Hackett G. Association of sexual problems with social, psychological, and physical problems in men and women: A cross-sectional population survey. J Epidemiol Community Health 1999;53:144-148.

25. Choi-Kwon S, Kim J. Post-stroke emotional incontinence and decreased sexual activity. Cerebrovasc Dis 2002;13:31-37.

26. Porpora M, Koninckx P, Piazze J, Natili M, Colagrande S, Cosmi E. Correlation between endometriosis and pelvic pain. J Am Assoc Gynecol Laparos 1999;6:429-434.

27. Wandell P, Brorsson B. Assessing sexual functioning in patients with chronic disorders by using a generic healthrelated quality of life questionnaire. Quality Life Res 2000;9: 1081-1092.

28. Wassertheil-Smoller S, Blaufox M, Oberman A, et al. Effect of antihypertensives on sexual function and quality of life: The TAIM Study. Ann of Intern Med 1991;114:613-620.

29. Grimm R, Grandits G, Prineas R, et al. Long-term effects on sexual function of five antihypertensive drugs and nutritional hygienic treatment in hypertensive men and women. Treatment of Mild Hypertension Study (TOMHS) Hypertension 1997;19:8-14.

30. Clayton A, Pradko J, Croft H, et al. Prevalence of sexual dysfunction among newer antidepressants. J Clin Psychiatry 2002;63:357-366.

31. Kennedy S, Mc Cann S, Masellis M, et al. Combining bupropion SR with venlafaxine, paroxetine, or fluoxetine: A preliminary report on pharmacokinetic, therapeutic, and sexual dysfunction effects. J Clin Psychiatry 2002;63:181-186.

32. Dennerstein L, Lehert $P$, Buirger $H$. The relative effects of hormones and relationship factors on sexual function of women through the natural menopausal transition. Fertil Steril 2005;84:174-180.

33. Bancroft J, Loftus J, Long J. Distress about sex: A national survey of women in heterosexual relationships. Arch Sex Behav 2003;32:193-208.

34. Hayes RD, Dennerstein L, Bennett CM, Koochaki PE, Leiblum SR, Graziottin A. Relationship between hypoactive sexual desire disorder and aging. Fertil Steril 2007;87:107-112.

35. Leiblum SR, Koochaki PE, Rodenberg CA, Barton IP, Rosen RC. Hypoactive sexual desire disorder in postmenopausal women: U.S. results from the Womens' International Study of Health and Sexuality (WISHeS) Menopause 2006;13:46-56.

36. Mitchell ES, Woods NF, Mariella A. Three stages of the menopausal transition: Toward a more precise definition. Menopause 2000;7:334-349.

37. Soules MR, Sherman S, Parrott E, et al. Executive summary: Stages of Reproductive Aging Workshop (STRAW). Fertil Steril 2001;76:874-878.

38. Harlow SD, Crawford S, Dennerstein L, Burger HG, Mitchell ES, Sowers MF, ReSTAGE Collaboration. Recommendations from a multi-study evaluation of proposed criteria for staging reproductive aging. Climacteric 2007;10:112-119.

39. Denari JH, Farinati Z, Casas PR, Oliva A. Determination of ovarian function using first morning urine steroid assays. Obstet Gynecol 1981;58:5-9.

40. Stanczyk FZ, Miyakawa I, Goebelsmann U. Direct radioimmunoassay of urinary estrogen and pregnanediol glucuronides during the menstrual cycle. Am J Obstet Gynecol 1980;137:443-450.

41. O'Connor KA, Brindle E, Shofer JB, et al. Statistical correction for nonparallelism in a urinary enzyme immunoassay. J Immunoassay Immunochem 2004;25:259-278.

42. Lasley BL, Shideler SE. Methods for evaluating reproductive health of women. Occup Med 1994;9:423-433.

43. Wilcox AJ, Baird DD, Weinberg CR, et al. The use of biochemical assays in epidemiologic studies of reproduction. Environ Health Perspect 1987;75:29-35.

44. Munro CJ, Stabenfeldt GH, Cragun JR, Addiego LA, Overstreet JW, Lasley BL. Relationship of serum estradiol and progesterone concentrations to the excretion profiles of their major urinary metabolites as measured by enzyme immunoassay and radioimmunoassay. Clin Chem 1991;37:838-844.

45. Robard D. Statistical quality control and routine data processing for radioimmunoassays and immunoradiometric assays. Clin Chem 1974;20:1255-1270.

46. Woods NF, Mitchell ES, Smith-DiJulio K. Cortisol levels during the menopausal transition and early postmenopause: Observations from the Seattle Midlife Women's Health Study. Menopause 2009;16:708-718.

47. Taussky HH. A microcolorimetric determination of creatinine in urine by the Jaffe reaction. J Biol Chem 1954;208:853-861.

48. Brantley PJ, Waggoner CD, Jones GN, Rappaport NB. A daily stress inventory: Development, reliability, and validity. J Behav Med 1987;10:61-74.

49. Pinheiro J, Bates D, DebRoy S, Sarkar D. nlme: Linear and nonlinear mixed effects models, $\mathrm{R}$ package version 3.1-66, 2005.

50. R Development Core Team. R: A language and environment for statistical computing. Vienna, Austria: R Foundation for Statistical Computing, 2005. Available at www.R-project.org 
51. Sarkar D. Lattice: Lattice Graphics. R package version 0. 12-11.

52. Pinheiro J, Bates D. Mixed-effects models in S and S-PLUS. New York: Springer, 2000.

53. Hox, J. Multilevel analysis: Techniques and applications. Mahwah, NJ: Lawrence Erlbaum Associates, 2002.

54. Gracia CR, Sammel MD, Freeman EW, Liu L, Hollander L, Nelson DB. Predictors of decreased libido in women during the late reproductive years. Menopause 2004;11:144-150.

55. West SL, D'Aloisio AA, Agans RP, Kalsbeek WD, Borisov NN, Thorp JM. Prevalence of low sexual desire and hypoactive sexual desire disorder in a nationally representative sample of US women. Arch Intern Med 2008:168:1441-1449.

56. Avis NE, Zhao X, Johannes CB, Ory M, Brockwell S, Greendale GA. Correlates of sexual function among multiethinic middle-aged women: Results from the Study of Women's Health Across the Nation (SWAN). Menopause 2005:12:385-398.

57. Reed SD, Newton KM, LaCroix AZ, Grothaus LC, Ehrlich K. Night sweats, sleep disturbance, and depression associated with diminished libido in late menopausal transition and early postmenopause: Baseline data from the Herbal Alternatives for Menopause Trial (HALT). Am J Obstet Gynecol 2007:196:593.e1-7.

58. Hayes RD, Dennerstein L, Bennett CM, Sidat M, Gurrin LC, Fairley CK. Risk factors for female sexual dysfunction in the general population: Exploring factors associated with low sexual function and sexual distress. J Sex Med 2008;5:16811693.

Address correspondence to: Nancy Fugate Woods, R.N., Ph.D. Department of Family and Child Nursing

University of Washington T318 Health Sciences Building

Box 357262

Seattle, WA 98195-7260

E-mail: nfwoods@u.washington.edu 